\title{
TOWARDS REAL-TIME CYBER-PHYSICAL SYSTEMS INSTRUMENTATION FOR CREATING DIGITAL TWINS
}

\author{
Joost Mertens, Moharram Challenger, Ken Vanherpen, Joachim Denil \\ Electronics-ICT Department, University of Antwerp, Belgium \\ Flanders Make, Leuven, Belgium \\ \{joost.mertens, joachim.denil, moharram.challenger, ken.vanherpen\}@uantwerpen.be
}

\begin{abstract}
One of the challenges with digital twins is data collection from the physical systems. Data collection for digital twins in Cyber-Physical Systems, where the controllers are often hard real-time, is a challenging task, as the functional system behavior may be impacted by the instrumentation. An often applied solution is the use of additional monitoring controllers, which passively monitor the system. However, this solution introduces additional points of failure, and is costly as more hardware is required. Therefore, in this paper, we propose an approach that aids the digital twin developer by finding the optimal instrumentation rate for a set of parameters that are to be instrumented on a hard real-time controller. The optimization ensures that the hard real-time constraints are guaranteed, while also taking into account the developer's preferences with regards to the instrumented parameters. We apply this process on two cases: an exploratory example and an adaptive cruise controller.
\end{abstract}

Keywords: Digital Twin, Cyber-physical Systems, Monitoring, Optimization, Instrumentation

\section{INTRODUCTION}

The term digital twin (DT), first coined in 2002 (Grieves 2016), has gained increasing interest in recent years due to the rise of industry 4.0 and industrial IoT. The digital twin concept was originally introduced for product life-cycle management. In the concept phase, a product design starts out as a digital prototype (Digital Twin Prototype or DTP), variants of this prototype are created/instantiated as physical products (Physical Twin or PT) and monitored by a digital instance (Digital Twin Instances or DTI). Changes in the Physical Twin propagate to the Digital Twin Instance through data transfer, and optimizations to the Physical Twin determined by the Digital Twin Instance propagate in the other direction. The aggregation of all DTIs is called a Digital Twin Aggregate (DTA), it is used to collect data from all instances, and can thus perform intelligent monitoring. For example if a PT fails, and the DTI logs the sensor data leading to that failure, this data can be used as a predictor for other equivalent physical devices (Grieves 2016). When the term Digital Twin is used, most often Digital Twin Instance and/or Digital Twin Aggregate is meant, e.g. "the digital twin of the machine" or "the digital twin of the factory".

Digital Twins offer various advantages, ranging from historical data logs, to more advanced possibilities such as preventive maintenance and process optimization. In all cases, the enabling source is the synchronization between the physical and digital worlds by communicating large amounts of data. The synchronization of both worlds is especially difficult when the DT must be synchronized in real-time. In Cyber-Physical Sys- 
tems (CPS), which are often hard-real time, this synchronization is also challenging, as none of the hard-real time constraints can be violated. In this regard, the rise of Internet-of-Things (IoT), and the accompanying sensing devices provides a solution to the synchronization problem. Instead of adding data collection on the hard real-time controller, data is collected by additional sensing devices. Using these sensing devices to monitor the physical system and transfer data to the DT is advantageous for two reasons: (1) the main system controller remains unaltered, and is unaffected by the monitoring devices, (2) the monitoring devices can be retrofitted on systems that only recently gained a DTI. Besides these advantages, there are also some disadvantages: firstly, the additional hardware introduces additional costs to the system. Secondly, each sensor node is an additional potential point of failure.

For existing systems, the passive instrumentation with additional sensors is advantageous, however for new systems, where the knowledge of the monitoring is already available at design time, the method of adding additional sensors is cumbersome and advantage (2) is negated. As an alternative to adding additional sensors, we propose that for new systems the main controllers also perform the monitoring and synchronization with the digital twin on top of their regular control functionality. Although the controller is now affected by the monitoring, the functional behavior can be guaranteed, as the system is still in its design phase, which still allows for relatively low-cost tweaking of the system. Additionally, the two disadvantages are now negated. The big challenge is ensuring that the functional behavior of the controller remains unaffected by the instrumentation. Numerous existing researches have already focused on program instrumentation on embedded systems (Fischmeister and Lam 2010),(Kashif and Fischmeister 2012), run-time monitoring on constrained systems (Medhat, Bonakdarpour, Kumar, and Fischmeister 2015),(Zheng, Julien, Podorozhny, Cassez, and Rakotoarivelo 2018) and instrumentation with preservation of extra-functional properties (Kashif, Arafa, and Fischmeister 2013), however, this knowledge has not yet been transferred to the data collection in digital twins. Nevertheless, there are shared elements between the two, mainly with regards to the real-time monitoring of devices.

In this paper, we propose an optimization method, that aids the DT developer by finding the optimal instrumentation rate for a set of parameters that are to be instrumented. The optimization ensures that the real-time behavior of the task model remains unchanged while taking into account the preferences of the system engineer with regards to the priority of the instrumented parameters. We apply our method on a simple example and on a case study of an adaptive cruise control (ACC) system.

The remainder of this paper is structured as follows: In section 2, we present the related work and give some background information. Next, in section 3 we elaborate the assumptions we made. Then, in section 4 we formulate and constrain the optimization problem. Thereafter, in sections 5 and 6 we discuss an exploratory example and the case study of an adaptive cruise control, on which we apply the method. Finally, in 7 we conclude the paper and discuss the future work.

\section{RELATED WORK}

We discuss the related work in two different areas: (a) Digital Twin research challenges, and (b) Program instrumentation.

\subsection{Digital Twin Challenges}

In (Grieves 2016), the concepts of PT, DTP, DTI and DTA are introduced and explained. In this study we cover these terms in section 1. In this section, we mainly discuss one of the challenges of digital twins for CPS, as presented in (Weyer, Meyer, Ohmer, Gorecky, and Zühlke 2016). This challenge is the synchronization between the real and the digital world, or in the terms of Grieves (2016), the PT and the DTI. Weyer, Meyer, Ohmer, Gorecky, and Zühlke (2016) note that for this synchronization tremendous volumes 
of data are needed, and the synchronization must be in real time for the DTI to serve its full purpose. The question that arises is how to monitor the PT to keep the DTI up to date. In (Zipper, Auris, Strahilov, and Paul 2018), active and passive monitoring are proposed as solutions to this problem. Passive monitoring is monitoring of the system where the original system remains unchanged, usually this is done by introducing additional devices. An example of passive monitoring in a CPS could be a monitoring device that sniffs data from a data bus. Active monitoring is a type of monitoring in which the existing controllers are affected by the monitoring. The controllers can be internally affected, for example by additional executable code for the monitoring, but also externally, for example a monitoring device could poll the controller for data. As stated in section 1, passive monitoring is advantageous, as it can be retrofitted to existing systems without influencing them. Also, it is an excellent use case for IoT devices, which are increasingly popular.

\subsection{Instrumentation}

Instrumentation is the act of modifying a program by adding instrumenting code to it. This code collects data during the program execution. Instrumentation can be either static, which means the instrumentation happens prior to the execution, and dynamic, which means the instrumentation happens at run-time. Instrumentation is possible at multiple levels: the binary executable level, the source code level or even higher levels, such as the model level. In general, instrumentation preserves the logical correctness of programs, however it does not always preserve extra-functional properties (for example the memory size).

Some research focuses specifically on preserving the extra-functional properties (Kashif and Fischmeister 2012, Kashif, Arafa, and Fischmeister 2013, Fischmeister and Lam 2010). In (Fischmeister and Lam 2010), Fischmeister and Lam (2010) propose an approach for instrumenting programs at the code level that preserves the logical correctness and the WCET. In (Kashif, Arafa, and Fischmeister 2013), the method is further refined to the INSTEP framework, which uses cost models and instrumentation intents to find an optimal solution. The instrumentation intent allows the developer to specify how the program should be instrumented. Another library for dynamic binary instrumentation can be found in (Arafa, Kashif, and Fischmeister 2013).

Although these techniques demonstrate practicality and scalability, they operate at the source code or binary level, and not at the model level, which makes them less suited for an Model Driven Engineering approach, as they are only applicable after code has been generated. Additionally, they do not take into account the task model of the software. A method more suited for the model driven approach is presented in (Denil, Kashif, Arafa, Vangheluwe, and Fischmeister 2015). The method we present here follows a similar approach to (Kashif, Arafa, and Fischmeister 2013), in that we also optimise based on instrumentation intent, cost models and extra-functional constraints, however it is slightly more generic in that it is not limited to code.

\section{PROPOSED APPROACH, MODELS, AND ASSUMPTIONS}

The objective of the instrumentation problem is to find an instrumentation of the model that fulfills the developers requests, and that maintains the functional correctness of the system. We specifically assume that causal-block diagrams are used, where each major block is implemented in a task on a real-time operating system. The implementation of the block-diagrams could for example be in Simulink (MathWorks 2020). We assume the real-time operating system uses a fixed priority preemptive scheduler. In this case it becomes clear that each task has a set of variables that the developer possibly wants to monitor. Some variables are more important than others, and additionally, not all variables need to be monitored at the same rate. As such, we allow the developer to specify for each variable a priority and a bounded monitoring rate. The priority ensures that higher priority variables are given a better rate than lower priority tasks, if the system is faced with multiple options. During execution, the system will have some slack time, which is time when 
the processor is unoccupied. Adding the monitoring of the variables introduces an additional cost in the execution time, i.e. it lengthens the execution time. As a result, the optimization that needs to happen is thus to instrument the model according to the developers request, optimally utilizing the slack time, but not breaching any deadlines of the tasks.

With regards to the digital twin for which the data is intended, we assume that there is no difference in the required data during initialization and steady state operation, as such the optimization is applicable for both cases. Additionally, we assume that the physical medium used to transmit the data suffices and is reliable, so, our optimization must not take into account re-transmission of the data.

\section{FORMULATION AND CONSTRAINTS}

We formulate the problem as a non-linear problem for which we have a set of variables, $V$, and a set of tasks, Tasks. The variables are constrained in their valid operating range. The tasks are constrained by their deadline, which must be attained in all cases. The solver of the problem tries to minimize an objective function, while adhering to the given constraints. The goal in this case, is to minimize the monitoring rate, $r_{v}$ of the variables. The monitoring rate, $r_{v}$, says that variable $v \in V$ is monitored once every $r_{v}$ task executions. As such, the monitoring rate of a variable, $v$, combined with the period of a task determines the period $T_{v, \text { monitor }}$ at which $v$ is monitored:

$$
T_{v, \text { monitor }}=r_{v} * T_{\text {task }_{i}}
$$

with $T_{v, \text { monitor }}$ the monitoring period of variable $v$ and $T_{\text {task }}$ the period of task $i$. The objective function to minimize is:

$$
\min \sum_{v \in V} r_{v} * p_{v}
$$

with $V$, the set of all variables to be monitored, $r_{v}$ the monitoring rate of variable $v$, and $p_{v}$ the priority of variable $v$, where a higher value of $p$ means the variable is more important. The next sections cover the constraints of the problem in more detail.

\subsection{Constraint 1: Cost of Monitoring the Variables}

Monitoring variables has multiple costs, for example: memory cost, temporal cost, binary size cost. In this study, we only consider the temporal cost, though the approach can be generalised to cover the other costs as well. Monitoring the variables has a fixed and a variable temporal cost. A fixed cost could be the initialization of a transmission buffer (once), whereas the variable cost could be the cost to fill the buffer with a variable value (once per variable). We assume that there is one fixed cost per rate at which a variable is monitored, per task in the RTOS. For each variable monitored at this rate we assume an additional variable cost. In a formula:

$$
C_{r, \text { monitoring }, \text { task }_{i}}=C_{f i x, r}+C_{v a r, r} * n_{r} .
$$

Or in words, the cost to monitor variables in a task $i$ with a certain rate $r$ consists of the fixed cost to monitor with this rate $C_{f i x, r}$ plus the variable $\operatorname{cost} C_{v a r, r}$ times the number of variables in this task monitored with this rate $n_{r}$. For now, we assume that the fixed and the variable cost are not influenced by the type of the variable being monitored, though it would be more correct to have the variable type influence the variable cost. 
For a task $i$ with multiple monitoring rates, the raw Worst-Case Execution Time (WCET), which is the raw computation time of this task, without possible preemption from other tasks, is given by:

$$
C_{\text {raw }, i}=C_{\text {base }, i}+\sum_{\forall r \in \text { task }} C_{r, \text { monitoring,task }}
$$

with $C_{\text {base }, i}$ the WCET without any monitoring, and $\forall r \in t_{a s} k_{i}$ meaning all the monitoring rates used in $\operatorname{task}_{i}$.

\subsection{Constraint 2: None of the Tasks may miss their Deadline}

Given the assumption of using an RTOS, the tasks run with deadlines, and their execution order is determined by their priorities. As such, the raw computation time does not suffice to check for deadline misses, and we must take into account the fact that the tasks can preempt each other, prolonging the execution time of the lower priority tasks. Due to the assumption of having only periodic tasks, we can calculate the WCET of the tasks, including the monitoring with the following formula (Joseph and Pandya 1986):

$$
C_{\text {real }, i}=C_{r a w, i}+\sum_{j \varepsilon H P(i)}\left\lceil\frac{C_{r e a l, i}}{T_{j}}\right\rceil * C_{r e a l, j}
$$

with $C_{\text {real }, i}$ the real WCET, taking into account scheduling, and $H P(i)$ the set of all tasks with a higher priority than task $i$. Here, it becomes clear that the problem at hand is not linear, as both $C_{r e a l, i}$ and $C_{r e a l, j}$ are variables, being multiplied. The only problem is that this WCET formula is overly pessimistic, as it disregards the rate at which the higher priority tasks are monitored, the result is that it essentially assumes that the computation times of the higher priority tasks is always their worst computation time, which would only be the case if the monitoring rate of all the variables is 1 . Therefore, we must alter the formula to make it less pessimistic:

$$
C_{\text {real }, i}=C_{r a w, i}+\sum_{j \in H P(i)}\left\lceil\frac{C_{r e a l, i}}{T_{j}}\right\rceil * C_{\text {base }, j}+\sum_{r \in R(j)}\left\lceil\frac{\frac{C_{r e a l, i}}{T_{j}}}{r_{j}}\right\rceil * C_{r, \text { monitoring }, \text { task }_{j}}
$$

where $R(j)$ is the set of all the monitoring rates used in $t a s k_{j}$. This formulation is still slightly too pessimistic, as it does not take into account phase shifts in the monitoring of the variables, but it is at least less pessimistic than the first formulation, and is less complex than attempting to also incorporate the phase shifts.

If each task $\mathrm{i}$ is then accompanied by a deadline $d_{i}$, we can state that

$$
\forall i \in \text { Tasks : } C_{\text {real }, i}<d_{i}
$$

\subsection{Constraint 3: the Possible Monitoring Periods}

The monitoring of the variables happens periodically, in a balanced way. We assume that the user provides the allowable monitoring rates as a range $[$ start,stop], this is a continuous range, which is not possible in the actual system. The constraint we impose on the monitoring rate is thus that it is an integer in this range: 


$$
r \in \mathbb{Z}_{>=1} \cup[\text { start }, \text { stop }] .
$$

The entire system will now have a hyperperiod, which is equal to:

$$
\text { HyperPeriod }=H P=l c m\left(\forall i \in \text { Tasks, } v \in V(i): T_{i} * r_{v}\right) .
$$

Or in words, the hyperperiod is equal to the least common multiple of a variable's monitoring rate times the tasks period, for all tasks and all variables.

\subsection{Optimization}

The problem formulation described above has a linear objective function, however it has a nonlinear constraint, constraint 2 . In this constraint, $C_{\text {real }, i}$ and $C_{r, \text { monitoring,task }}$ are multiplied, but they are both variables, yielding a nonlinear problem. The proper and more scalable way to solve this problem would be to apply a nonlinear solver, however solving this problem for a global minimum would be fairly computationally expensive. Instead, we will apply a least-cost branch and bound algorithm. For this algorithm, we require a bounding function, which bounds the search space by estimating the remaining cost of the future steps of the algorithm. In this case, the bounding function operates as follows: for each of the tasks, we know the lower thresholds for the monitoring rate of its variables, as such, for each level of the tree, we can precompute the lowest theoretical cost. With these theoretically lowest costs, we can precompute for each level what the theoretical minimal partial cost of the remaining levels is. The bounding function then checks the following:

partial cost of current path + sum(minimum cost of all remaining levels $)<=$ lowest cost .

Or thus, it checks if the partial cost of the current path plus the partial cost of the remaining levels is smaller than the current lowest cost. If it is, the branch is worth exploring further, as with the theoretical minimum, it might be possible to find a new solution. If not, this branch is not worth exploring further.

The algorithm to optimize the objective function is as follows:

1. Initialize the lowest cost to infinity. Precompute the remaining partial costs needed for the bounding function.

2. Begin the branching tree at the root node. The children of the root level are all possible combinations of monitoring rates for the highest priority task. These children are sorted, such that the children with the lowest partial cost (= the objective function, but only applied to this child) are at the left side of the tree. The children for which the WCET constraint does not hold true can be immediately pruned.

3. Each of these children yields one solution for the variable $C_{r, \text { monitoring,task }}$, which can be applied in the calculation of $C_{\text {real }, i}$ for the lower priority tasks.

4. Starting with the leftmost child (= the child with the lowest partial cost), consider it as the current node, then perform the bounding check. It is worth to note that the bounding check will be applied twice in the iteration, this is by design. The bounding check in this step is because at the beginning, no solution has been found yet and the bounding check will pass for all of the initial branches. Later, when the algorithm backtracks to the initial branches and a solutions has been found, it could be that the bounding check fails, and these branches can thus be skipped. If it passes, continue with step 5 . If it fails, attempt to move up to a sibling of the parent of the current node in the tree (or if this parent has no sibling, however many parents higher in the tree until a sibling is found or the tree is finished 
traversing). The reason we can do this is because the tree is ordered from left to right, as such, if a node fails the bounding check, all its higher cost siblings will also fail the bounding check.

5. calculate all the combinations of monitoring rates for the task with a priority one level lower. Prune all the combinations for which the WCET constraint does not hold. For the combinations for which the WCET constraint does hold, calculate the bounding function for that combination on the next level. Prune all those combinations for which the bounding check fails. Essentially this is step 2, but for one of the branches instead of the root node.

6. The remaining combinations all pass the bounding check so they can be added to the tree as children of the current node. If the added children correspond to the lowest priority task (= leaf nodes of the tree), calculate the current total path cost using the child with the lowest partial cost. We consider this path as one of the solutions for the problem. The cost of this path is now the lowest cost, any other solution must either have an equal or lower cost than the current lowest cost. Next, check if any of the siblings of the current child with the lowest cost have an equal cost, these children can also be added to the solution. If no combinations remained, no children can be added, and the current node should move to its leftmost sibling (or if this node has no sibling, try to move to a sibling of the parent node, or if this parent has no sibling, however many parents higher in the tree until a sibling is found or the tree is finished traversing).

7. repeat steps 4-6 until either the requested number of solutions is found, or the tree is exhausted, which means the current node was moved back to the root node, either through the move when the bounding check in step 4 fails, or through the move in step 6 .

\subsection{Discussion of Drawbacks of the Optimization Method}

Linear problems, often integer, are solvable with MILP solvers. The use of a MILP solvers ensures that a global optimum can be found. In this study, the presented problem is linear, but one of the constraints is not, which makes the problem non-linear, which is why we cannot solve it with a MILP solver. The proposed optimization tries to solve the problem through the heuristic method explained in the previous section. This method currently has multiple drawbacks, which require further investigation in the future:

- The user must request an appropriate number of solutions: One of the stop conditions of the algorithm is the number of found solutions. The problem with this parameter is as follows: if the user picks a small value, it is possible that not all the solutions are found. If however, the user picks a large number, it is possible that this many solutions do not exist. As a result the algorithm will run until the tree is exhausted, which can take a significant amount of time, even with the pruning.

- Task and parameter priorities are implicitely linked for a small number of solutions: The idea of parameter priorities is for the engineer to indicate which parameters should be prioritized to get a low monitoring rate. Because our linearization method forces us to find solutions starting with tasks at a higher priority, the parameters of high priority tasks are at an advantage when a limit is placed on the number of requested solutions. In general, the first solutions the algorithm finds are solutions where the variables of the higher priority tasks have a lower rate. As such, when only a small number of solutions are requested, these solutions are found first. In the ideal case, the parameter and task priorities are completely decoupled, as it is possible that low priority tasks have high priority parameters.

- Massive search space: The algorithm attempts to find the best solutions, by handling the lower cost partial solutions first, however, this does not put a bound on the number of partial solutions. For each task, all the possible combinations of parameter monitoring are generated and ranked by their cost. For tasks with little parameters and limited parameter monitor rate intervals, this combination is feasible, however when either of those two variables becomes large, the number of combinations 
quickly grows massively. Although the number of combinations is pruned by the constraints, it is still a very large number. When a large range of monitoring intervals is specified, a possible solution is to focus on the subset of rates with the lowest cost first, and only expand this subset when no viable solutions are found. For tasks with many parameters, we could potentially offer the possibility for optional variables, that could reduce the search space.

While some of these drawbacks still offer ways reduce their impact, we think that in the future we should instead focus on using a non-linear problem solver. In this case, the problem has a couple of advantages, in that the solution is integer, and the permissable range of the variables is constrained within a limited range. As such, we believe that reformulating the problem is a solver such as Mystic (McKerns, Strand, Sullivan, Fang, and Aivazis 2011) is a viable alternative, which may yield faster results.

\section{EXPLORATORY EXAMPLE}

To gain more insight into how the solver works, we first explore a small example that one could still solve manually. The example also demonstrates some of the drawbacks of the method. The problem formulation is given in Table 1. We note for example that all variables have an equal priority, and that there is no fixed cost for monitoring, this could be the case if this cost is sufficiently small compared to the execution time. Note that the problem can be considered unit-less, but for simplicity, we assume all time units are in ms, so, for example, the tasks T1 and T2 have periods of 4 and $6 \mathrm{~ms}$.

Table 1: Exploratory problem formulation. With $\mathrm{T}$ the task period, $\mathrm{d}$ the task deadline and $\mathrm{p}$ the task or variable priority, $\mathrm{r}_{l t h}$ and $\mathrm{r}_{u t h}$ respectively the lower and upper thresholds for the allowed instrumentation rates.

\begin{tabular}{|c|c|c|c|c|c|c|c|c|c|c|}
\hline & & & & & Variable & $\mathrm{r}_{l t h}$ & $\mathrm{r}_{u t h}$ & $\mathrm{p}$ & \multirow{7}{*}{$\begin{array}{l}\text { Fixed cost } \\
\text { Variable cost }\end{array}$} & \\
\hline & & & & & T1 V1 & 1 & 5 & 1 & & \\
\hline Task & $\mathrm{T}$ & $\mathrm{C}_{\text {base }}$ & d & $\mathrm{p}$ & T1 V2 & 1 & 5 & 1 & & \\
\hline $\mathrm{T} 1$ & 4 & 1 & 4 & 2 & T1 V3 & 1 & 5 & 1 & & \\
\hline \multirow[t]{3}{*}{$\mathrm{T} 2$} & 6 & 1 & 6 & 1 & $\mathrm{~T} 2 \mathrm{~V} 1$ & 2 & 6 & 1 & & 0.4 \\
\hline & & & & & $\mathrm{T} 2 \mathrm{~V} 2$ & 2 & 6 & 1 & & \\
\hline & & & & & $\mathrm{T} 2 \mathrm{~V} 3$ & 2 & 6 & 1 & & \\
\hline
\end{tabular}

The easiest way to solve this exploratory example manually is by drawing the timeline of the tasks. An example is given in Figure 1. We assume that both tasks are activated at $t=0$. Looking at the periods, the cost of monitoring and the deadlines, we can see that for T2, the variables can be monitored at their lowest allowed rate, which is 2 (so every other execution is monitored). For T1, which has a higher priority than $\mathrm{T} 2$, one of the variables can be monitored at its lowest rate, which is 1 , and the other two must be monitored at a rate of 2. A lower rate is not possible, as otherwise the first activation of T2 would not meet its deadline. In the example timeline, T1V3 is monitored at a rate of 1 and T1V1 and T1V2 are monitored at a rate of 2. Since these variables have the same priority, we know that two other solutions exist as well, where either $\mathrm{T} 1 \mathrm{~V} 1$ or T1V2 is the variable with a rate of 1 instead of T1V3.

We now solve this problem with the solver. We solve for a requested number of solutions equal to $1,3,4$ and 6 and time how long the execution of the solver takes. The timing information is listed in Table 2. The timing was performed using the Python timeit module, on Anaconda Python 3.7, on an AMD Ryzen 51600 processor with $16 \mathrm{~GB}$ of RAM. In the timing table, we note that the results are as expected. Solving for only 1 solution takes the least amount of time, at $90 \mathrm{~ms}$. Solving for 3 solutions takes slightly longer, at $131 \mathrm{~ms}$. solving for more than 3 solutions, in this case 4 or 6 takes approximately the same amount of time, as the tree is now being traversed completely. The program then takes the longest path, resulting in an execution 


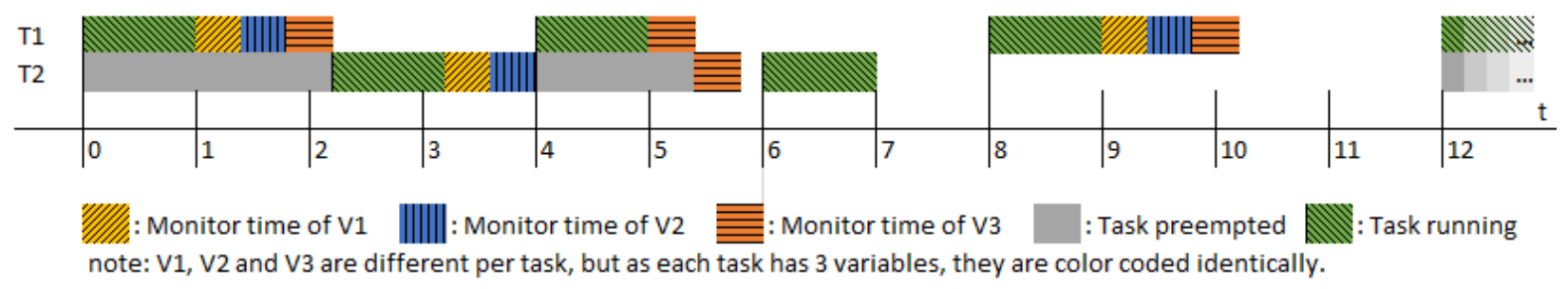

Figure 1: Timeline of one of the possible solutions of the exploratory problem.

time of 0.696 and 0.703 seconds, which can be considered equal. This is 5.3 times longer than when 3 solutions are requested and 7.8 times longer than when only 1 solution is requested. This again shows the disadvantage of requiring the user to request an appropriate amount of solutions. The developer must thus always make a judgment call on the number of requested solutions. Request too little solutions and not all solutions will be found, request too many and the program now takes a long time to solve.

Table 2: Computation time for different numbers of requested solutions.

\begin{tabular}{r|cccc} 
Requested number of solutions & 1 & 3 & 4 & 6 \\
\hline Computation time [s] & 0.090 & 0.131 & 0.696 & 0.703
\end{tabular}

The solutions are given in Table 3, and show what was expected: for 1 requested solution, only the first solution is shown, for 3 all 3 possible solutions are shown, and for 6 only 3 solutions are shown, as there are only 3 solutions with the lowest cost. The lowest cost in this case is 11 , which is logical as $2 * 5+1 * 1=11$. From the verbose logging, we can see that other solutions that adhere to the constraints exist as well, but they all have a higher cost than 11, and are thus not returned.

Table 3: Solutions provided for the exploratory problem.

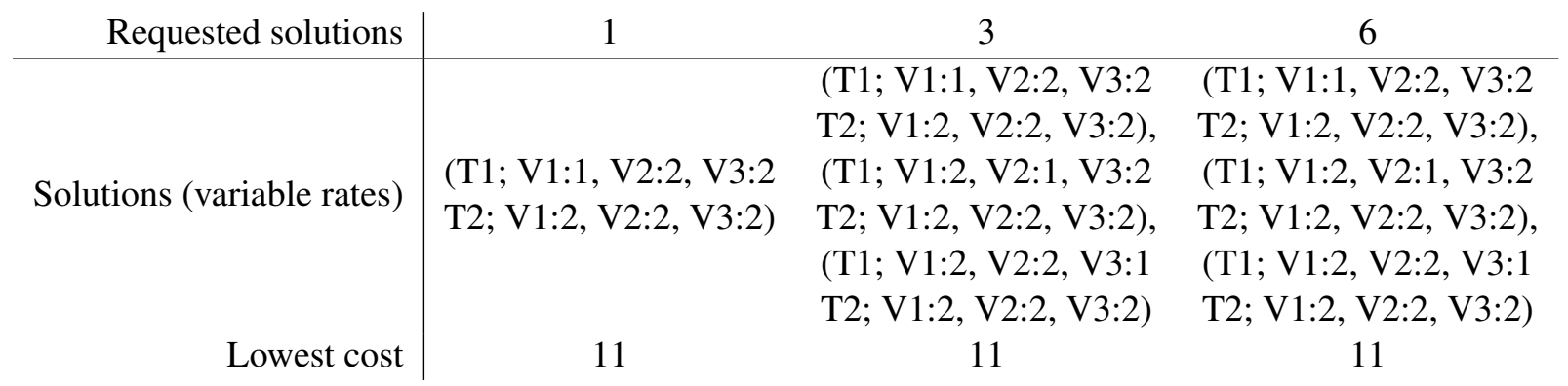

\section{CASE STUDY: ADAPTIVE CRUISE CONTROL}

While the exploratory example is a fine problem, as one could still solve it manually, it is fairly simple. Therefore, we also conducted some experiments on an adaptive cruise controller (ACC) system. Note that we currently do not have measured execution times of the system, as such, all WCET times are only estimates, as are the monitoring costs. Nonetheless, the ACC system still provides a more practical example than the exploratory problem. In what follows we discuss the system and the achieved results.

\subsection{System Overview}

The system in this case study is an ACC controlling an Radio Controlled (RC) car (O'Kelly, Sukhil, Abbas, Harkins, Kao, Pant, Mangharam, Agarwal, Behl, Burgio, and Bertogna 2019). A schematic overview of the system is given in Figure 2. The system consists of three tasks: one communication task, for communication 
with the transmitter, one car controller task, which handles the control of the car and monitors variables such as the current speed, and one ACC task, which performs the ACC calculations when the ACC is enabled. The system is made with responsiveness in mind, as such, the Communication Task has the highest priority, the Car Controller Task the next highest priority, and the ACC Task the lowest priority. We already encounter one obstacle, which is that the ACC task can be enabled or disabled, which does not conform to the assumption of a system with purely periodic tasks. For the further discussion, we simply assume the ACC to be enabled at all times.

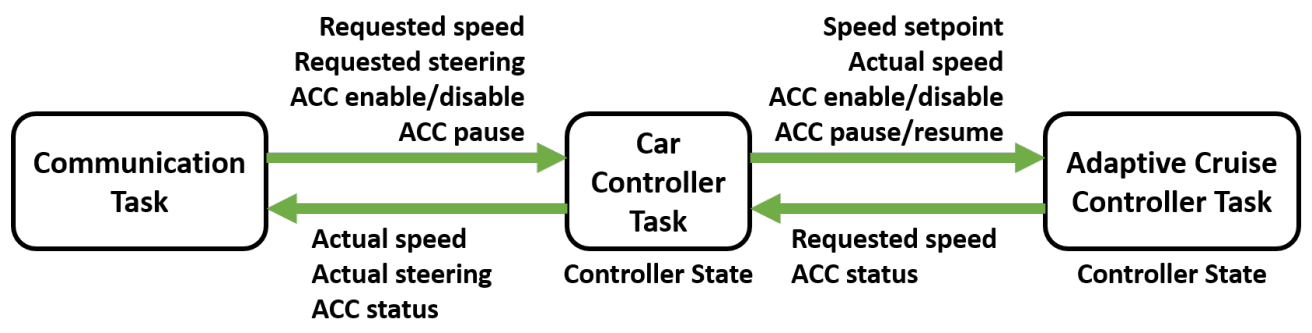

Figure 2: Overview of the tasks and data communication between them in the ACC.

\subsection{Problem Formulation}

The problem is formulated in Table 4. All time units in the problem formulation are in ms. With regards to the data being monitored, we note that as the data is shared, some variables can technically be monitored in two different tasks. In this case, we simply monitor the data at the most appropriate location. For example, the Car Controller Task monitors the actual speed of the car. It shares this data with both the Communication and ACC Task, but as it is the main handler of this data, we monitor this variable in the Car Controller Task. As stated earlier, the WCET, and the costs of monitoring are only estimates.

Table 4: Problem formulation of the ACC case study.

\begin{tabular}{|c|c|c|c|c|c|c|c|c|c|c|c|}
\hline & & & & & Task & Variable & $\mathrm{r}_{l t h}$ & $\mathrm{r}_{\text {uth }}$ & $\mathrm{p}$ & & \\
\hline & & & & & Comm. & Req. speed & 1 & 5 & 3 & & \\
\hline & & & & & & Req. steering & 4 & 10 & 1 & & \\
\hline & & & & & & $\mathrm{ACC}$ en/dis & 4 & 10 & 1 & & \\
\hline Task & $\mathrm{T}$ & $\mathrm{C}_{\text {base }}$ & d & $\mathrm{p}$ & & ACC pause/res & 4 & 10 & 1 & & \\
\hline Comm. & 20 & 1 & 20 & 3 & Car Control & Actual speed & 1 & 5 & 3 & $\mathrm{C}_{\text {fixed }}$ & 0.2 \\
\hline Car Control & 10 & 3 & 10 & 2 & & Actual steering & 4 & 10 & 1 & $\mathrm{C}_{v a r}$ & 0.1 \\
\hline $\mathrm{ACC}$ & 8 & 2 & 8 & 1 & & Internal state & 2 & 5 & 2 & & \\
\hline & & & & & $\mathrm{ACC}$ & Speed setpoint & 4 & 10 & 1 & & \\
\hline & & & & & & Status & 1 & 5 & 3 & & \\
\hline & & & & & & Req. speed & 1 & 5 & 3 & & \\
\hline & & & & & & Internal state & 2 & 5 & 2 & & \\
\hline
\end{tabular}

\subsection{The Solution}

For the discussed problem, we found a solution, with a computation time of 56.6 seconds. The solution specifies that all the parameters have a rate of 4 , except for the requested speed parameter in the communication task, which has a rate of 1 . In this case, it seems that the fixed cost and the tight slack time forces the 
algorithm to use the same rate for a lot of the variables (recall that the fixed cost is per rate, so using more rates introduces more fixed cost).

\section{CONCLUSION AND FUTURE WORK}

In this study, we address the problem of parameter monitoring in hard real-time systems, which is related to the data collection required for the synchronization of digital twins. We also looked at how this problem is tackled in a field such as run-time monitoring on embedded systems. We then formulated a branchand-bound algorithm that can solve the non-linear problem at hand. We demonstrated a small exploratory example, to provide more insight in how the solver operates, and we also applied the solver on a slightly more complex case study of an adaptive cruise controller. The exploratory example showed some of the drawbacks of the solver, and gave more insight in its operation, whereas the ACC case study showed that more difficult problems can also be solved.

A lot of potential future directions exist. The logical next step would be to incorporate the algorithm in a model based design workflow, where using model transformations, the monitoring blocks are automatically added to the Simulink model, and configured with the rates found in the solution. Another step is to further elaborate on the ACC case study. In the current study, all the timing information is based on estimations, however for a proper solution, these timings should be measured. This can be done with RapiTime (RapitaSystems 2020), which is the de-facto standard for WCET analysis. Another future work, as alluded to in the Introduction, is to build a digital twin using the ACC system, on which we perform process monitoring. This is a fairly big leap and will require work in other areas as well, especially with regards to DT initialization, synchronization, and reliability. Recall that in the assumptions we stated that we assume there is no difference between the initialization and steady-state phase of the DT. This is an assumption that must be verified. Most likely, different data is needed during the initialization of the DT, than during steady-state operation. Additionally, recall that we assumed the network to be reliable, which is unlikely. In this area, studies such as (Han, Natale, Zeng, Liu, and Dou 2013) might give valuable insights. Lastly, we believe that the presented problem can also be solved by constrained non-linear problem solvers, such as Mystic (McKerns, Strand, Sullivan, Fang, and Aivazis 2011). Re-implementing the problem in such a solver, and comparing the solutions with the presented approach is another next step. Besides building on top of this work, we could also make a step to the side, and research instrumentation where the data is collected in batches. In such case, additional tasks are introduced, but the real-time constraints of those tasks are less stringent.

\section{ACKNOWLEDGMENTS}

This research was supported by Flanders Make, the strategic center for the manufacturing industry within the framework of the DTDesign ICON project.

\section{REFERENCES}

Arafa, P., H. Kashif, and S. Fischmeister. 2013. "DIME: time-aware dynamic binary instrumentation using rate-based resource allocation". In Proceedings of the Eleventh ACM International Conference on Embedded Software, pp. 25. IEEE Press.

Denil, J., H. Kashif, P. Arafa, H. Vangheluwe, and S. Fischmeister. 2015. "Instrumentation and preservation of extra-functional properties of simulink models". In Proceedings of the Symposium on Theory of Modeling \& Simulation: DEVS Integrative M\&S Symposium, pp. 47-54. Society for Computer Simulation International.

Fischmeister, S., and P. Lam. 2010, 12. "Time-Aware Instrumentation of Embedded Software". Industrial Informatics, IEEE Transactions on vol. 6, pp. $652-663$. 
Grieves, M. 2016, 08. “Origins of the Digital Twin Concept”. In Working Paper, pp. 1-7. Florida Institute of Technology.

Han, G., M. D. Natale, H. Zeng, X. Liu, and W. Dou. 2013. "Optimizing the implementation of real-time Simulink models onto distributed automotive architectures". Journal of Systems Architecture vol. 59 (10, Part D), pp. 1115 - 1127.

Joseph, M., and P. Pandya. 1986, 01. "Finding Response Times in a Real-Time System". The Computer Journal vol. 29 (5), pp. 390-395.

Kashif, H., P. Arafa, and S. Fischmeister. 2013. "INSTEP: A static instrumentation framework for preserving extra-functional properties". In 2013 IEEE 19th International Conference on Embedded and Real-Time Computing Systems and Applications, pp. 257-266. IEEE.

Kashif, H., and S. Fischmeister. 2012. "Program transformation for time-aware instrumentation". In Proceedings of 2012 IEEE 17th International Conference on Emerging Technologies \& Factory Automation (ETFA 2012), pp. 1-8. IEEE.

MathWorks 2020. "Simulink - Simulation and Model-Based Design".

McKerns, M. M., L. Strand, T. Sullivan, A. Fang, and M. A. G. Aivazis. 2011. "Building a Framework for Predictive Science”. ArXiv vol. abs/1202.1056, pp. 1-12.

Medhat, R., B. Bonakdarpour, D. Kumar, and S. Fischmeister. 2015. "Runtime Monitoring of CyberPhysical Systems Under Timing and Memory Constraints". ACM Transactions on Embedded Computing Systems (TECS) vol. 14, pp. 79.

Matthew O'Kelly and Varundev Sukhil and Houssam Abbas and Jack Harkins and Chris Kao and Yash Vardhan Pant and Rahul Mangharam and Dipshil Agarwal and Madhur Behl and Paolo Burgio and Marko Bertogna 2019. "F1/10: An Open-Source Autonomous Cyber-Physical Platform".

RapitaSystems 2020. "RapiTime I Rapita Systems".

Weyer, S., T. Meyer, M. Ohmer, D. Gorecky, and D. Zühlke. 2016. "Future Modeling and Simulation of CPS-based Factories: an Example from the Automotive Industry". IFAC-PapersOnLine vol. 49 (31), pp. 97-102. 12th IFAC Workshop on Intelligent Manufacturing Systems IMS.

Zheng, X., C. Julien, R. Podorozhny, F. Cassez, and T. Rakotoarivelo. 2018, June. "Efficient and Scalable Runtime Monitoring for Cyber-Physical System”. IEEE Systems Journal vol. 12 (2), pp. 1667-1678.

Zipper, H., F. Auris, A. Strahilov, and M. Paul. 2018, 02. "Keeping the digital twin up-to-date - Process monitoring to identify changes in a plant". In International Conference on Industrial Technology (ICIT), pp. 1592-1597. IEEE.

\section{AUTHOR BIOGRAPHIES}

JOOST MERTENS PhD student and teaching assistant at the University of Antwerp, Belgium. His email address is joost.mertens@uantwerpen.be.

MOHARRAM CHALLENGER A postdoc researcher at the University of Antwerp, Belgium. His email address is moharram.challenger@uantwerpen.be.

KEN VANHERPEN A postdoc researcher at the University of Antwerp, Belgium. His email address is ken.vanherpen@uantwerpen.be.

JOACHIM DENIL A professor at the University of Antwerp, Belgium. His email address is joachim.denil@uantwerpen.be. 